\title{
NKILA: A New LncRNA Regulates the Pro-inflammation Functions of NF-кB
}

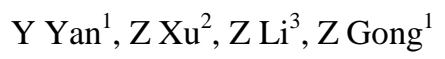

\section{INTRODUCTION}

Long non-coding RNAs (lncRNAs), a new class of non-coding RNAs longer than 200 nucleotides, have been recognized to regulate a wide variety of physiological and pathological processes through diverse mechanisms. Presently, only a small number of lncRNAs have been characterized functionally, and most of them are shown to act as regulators at different levels of gene expression $(1,2)$. Studies have revealed that many lncRNAs are shown to regulate important cancer hallmarks, including initiation, growth, and metastasis (3). However, whether lncRNA plays a role in cancer related inflammation is not clear yet. Recently, in Cancer Cell, Liu et al. reported a novel lncRNA upregulated by NF-кB (nuclear factor kappaB) was involved in cancer related inflammation by

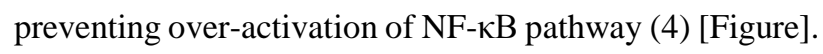

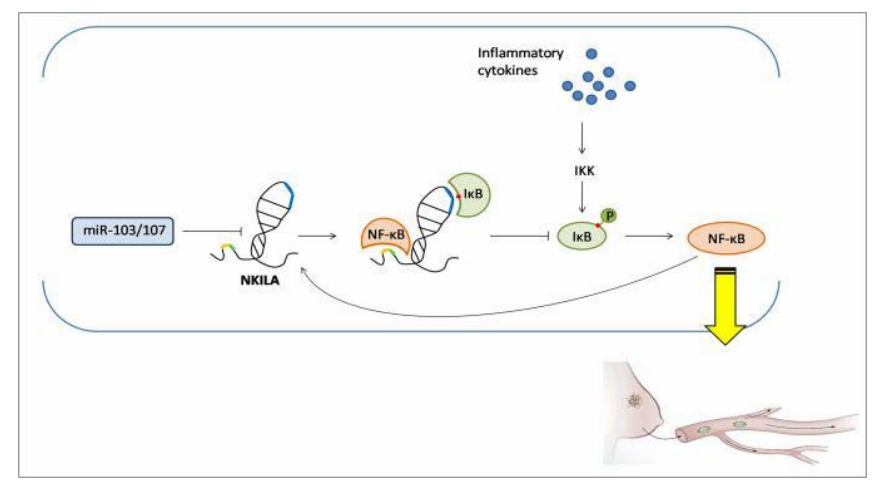

Figure: LncRNA NKILA acts as an interactor in the negative feedback loop of NF- $\kappa \mathrm{B}$ regulation, co-operating in the breast cancer metastasis. Activating NF- $\mathrm{B}$ signaling by inflammation promote NKILA expression, which in turn binds to NF- $\kappa \mathrm{B} / \mathrm{I} \kappa \mathrm{B}$, and directly masks phosphorylation motifs of $\mathrm{I} \kappa \mathrm{B}$, thereby inhibiting IKK-induced

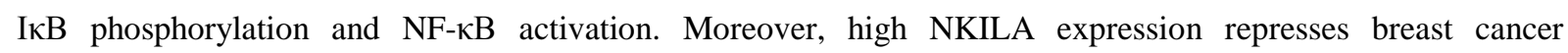
metastasis by preventing over-activation of NF- $\mathrm{B}$ pathway.In model of NKILA, yellow lines denotes hairpin A, green lines denotes hairpin $\mathrm{B}$, and blue lines denotes hairpin $\mathrm{C}$.

Keywords: Cancer, inflammation, IncRNA,nf-Kb

From: ${ }^{1}$ Department of Pharmacy, Xiangya Hospital, Central South University, Changsha, Hunan, P R China, ${ }^{2}$ Cancer Research Institute, Central South University, Changsha, Hunan, P R China, ${ }^{3}$ Center for Molecular Medicine, Xiangya Hospital, Central South University, Changsha, Hunan, P R China.

Correspondence: Dr Z Gong, Department of Pharmacy, Xiangya Hospital, Central South University, 87 Xiangya Road, Changsha, China, 410078. Email: gongzhicheng2013@163.com 
Aiming to identify lncRNAs involved in activating NF-кB signaling elicited by inflammation, Liu et al. profiled lncRNA expression in human breast cancer cells after treatment with inflammatory cytokines TGF- $\alpha$

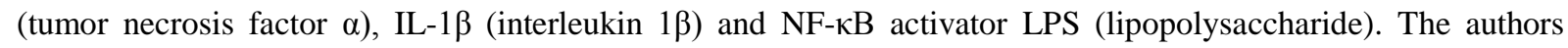
focused on the lncRNAs that could up- or down-regulate by all the above stimuli by more than 10-fold. One such lncRNA, aptly named NF-кB Interacting LncRNA (NKILA), stood out by virtue of up-regulating about 12-fold.

Furthermore, a constitutively active IkappaB kinase-2 (CA-IKK2), one member of NF-kB

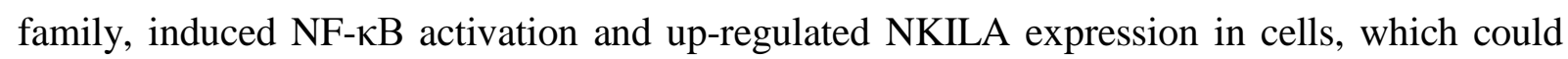
be completely inhibited by Sc-3060 and JSH-23, two inhibitors for NF- $\kappa$ B nuclear translocation. These findings suggest that NKILA may represent an essential node in the implement of pro-inflammation functions by NF-кB.

The authors then asked the mechanisms by which NKILA interfere NF- $\kappa \mathrm{B}$ activation. The cytoplasmic localization of NKILA by confocal microscopy implied that the lncRNA

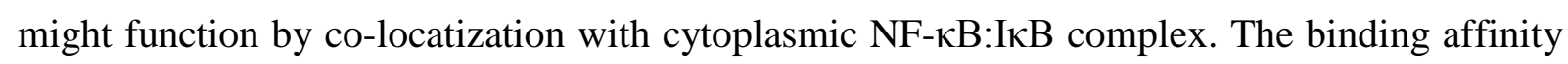
between NKILA and p65 was further confirmed by RNA immunoprecipitation (RIP). Two independent sets of software, Mfold and RNAfold, predicted nt 300-500 of NKILA, named hairpin A (nt 322-359) and hairpin B (nt 395-418), were essential for NKILA to bind p65 and

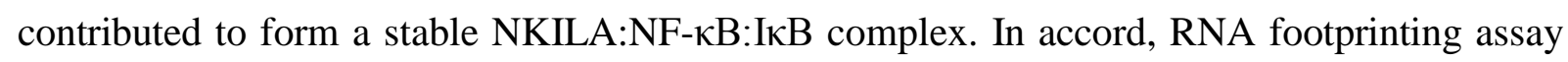
identified two different sites within hairpin A and hairpin B. Likewise, a triple-loop hairpin structure at nt 1121-1216 of NKILA (hairpin C), predicted by Mfold and RNAfold was further verified by RNA structure probing. The authors showed that NKILA directly interact with $\mathrm{I} \kappa \mathrm{B} \alpha$ and mask the phosphorylation motif of $\mathrm{I} \kappa \mathrm{B} \alpha$, which consequently inhibit IKKinduced I $\mathrm{B}$ phosphorylation. In addition, mutation assays at each of the hairpins 


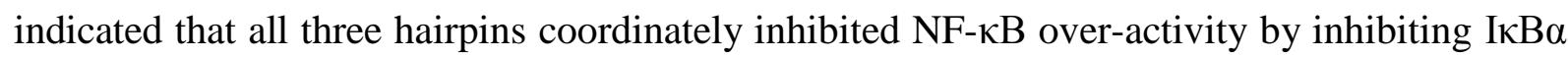
phosphorylation. These results indicated that NKILA is involved in the negative feedback loop of NF- $\kappa$ B regulation.

An interesting follow-up question is whether NKILA is degraded by specific microRNAs (miRNAs) via the 5'-to-3' messenger RNA (mRNA) decay pathway, i. e. after shortening of the mRNA poly(A) tail, the removal of the 5' cap structure by deadenylation triggers irreversible decay of the mRNA body (5).To address this issue, Liu and colleagues screened a comprehensive library of 434 miRNAs predicted to target NKILA by RegRNA in highmetastatic breast cancer cells. Through this analysis, the authors identified two miRNAs, miR-103 and miR-107 that were responsible for rapid degradation of NKILA. Remarkably, depletion of either miRNA by antisense oligonucleotides is sufficient to increasing NKILA expression. This decay effect could be restored by silencing key mediators for miRNAmediated RNA degradation, such as EIF2C2 (argonaute RISC catalytic component 2) and TRNC6A (Trinucleotide Repeat Containing 6A). miRNA is well-known to interact with LncRNAs (6) and the authors found a miR-103/107 target site at 1203-1234 nt of NKILA. Luciferase reporter assay using pMIR-REPORT constructs showed that co-transfection of miR-103 or miR-107 significantly reduced luciferase activity of the reporter gene. Taken together, these results demonstrated that miR-103 and miR-107 function to control RNA degradation, which offset the enhanced transcription of NKILA caused by high NF-kB activity.

It's now accepted that abnormal constitutively activation of NF- $\kappa B$ signaling pathway has a crucial role in inflammation, and further to promote cancer initiation and progression (7, 8). NF- $\mathrm{BB}$ inhibition is expected to be beneficial in the treatment of breast cancer (9). Liu et al. 
has demonstrated that NKILA effectively restrained breast cancer metastasis and improve clinical outcome by dramatically repressing NF-kB activities and cancer-associated inflammation. In addition, the association of reduced NKILA expression with cancer tissues across tissue types and the strong clinical significance of NKILA in breast cancer argue its potential utility as a therapeutic target. However, so far owing to the incomplete understanding of their function under specific circumstances, it is highly debatable to consider the application of lncRNAs as potential targets for the design of therapeutic agents currently (10) In the case of NKILA, it is intriguing that its lower expression is associated with only invasive rather than non-invasive malignant tissues given the pivotal role of NF- $\kappa \mathrm{B}$ in many facet of cancer-related-inflammation. Furthermore, Liu et al. reported the universal downregulation of NKILA in malignant prostate, colon and lung. In consideration of these two points, it is inferable that NKILA may preserve distinct roles in the context of tissue. In this regard, it may be helpful to investigate whether NKILA is associated with other facet of carcinogenesis rather than invasion and metastasis. Specifically, NKILA may promote malignance via other mechanisms involving RNA-protein-DNA or RNA-RNA interactions.

Recent publications have shown lncRNAs regulate important aspects of immunity such as production of inflammatory mediators, differentiation, and cell migration through RNA-protein interactions or basepair complement with RNA and DNA in inflammationrelated diseases (11). Despite the rapid development with the technologies associated with lncRNAs such as RNA-pull down, capture hybridization analysis of RNA targets (CHART) (12) and massive parallel sequencing, it is still challenging to identify functionally relevant lncRNAs due to obstacles including poor evolutionary sequence conservation, lack of animal model conservation across species and complex function of 
lncRNAs themselves involved. Regardless of these limitation, given the comprehensive and profound role of inflammation in pathology and carcinogenesis, it is reasonable to envisage that there are many additional inflammation-related lncRNAs to be discovered and further studies are required to explore the actual regulation mechanisms of lncRNAs, which will benefit to discover the new target for cancer therapy and accelerate the process of "bench to bed”.

\section{ACKNOWLEDGEMENTS}

The study was supported by the National Natural Science Foundation of China (81572946). There is no conflict of interests to declare. 


\section{REFERENCES}

1. Wahlestedt C. Targeting long non-coding RNA to therapeutically upregulate gene expression. Nat Rev Drug Discov 2013; 12: 433-446.

2. Batista PJ, Chang HY. Long noncoding RNAs: cellular address codes in development and disease. Cell 2013; 152: 1298-1307.

3. Ling H, Vincent K, Pichler M, Fodde R, Berindan-Neagoe I, Slack FJ et al. Junk DNA and the long non-coding RNA twist in cancer genetics. Oncogene 2015.

4. Liu B, Sun L, Liu Q, Gong C, Yao Y, Lv , et al. A cytoplasmic NF-kappaB interacting long noncoding RNA blocks IkappaB phosphorylation and suppresses breast cancer metastasis. Cancer Cell 2015; 27: 370-81.

5. Nishihara T, Zekri L, Braun JE, Izaurralde E. miRISC recruits decapping factors to miRNA targets to enhance their degradation. Nucleic Acids Res 2013; 41: 8692-705.

6. Wang P, Ning S, Zhang Y, Li R, Ye J, Zhao Z, et al. Identification of lncRNA-associated competing triplets reveals global patterns and prognostic markers for cancer. Nucleic Acids Res 2015; 43: 3478-89.

7. Hoesel B, Schmid JA. The complexity of NF-kappaB signaling in inflammation and cancer. Mol Cancer 2013; 12: 86.

8. Kravtsova-Ivantsiv Y, Shomer I, Cohen-Kaplan V, Snijder B, Superti-Furga G, Gonen H, et al. KPC1-mediated ubiquitination and proteasomal processing of NF-kappaB1 p105 to p50 restricts tumor growth. Cell 2015; 161: 333-47.

9. Liu R, Liu C, Chen D, Yang WH, Liu X, Liu CG et al. FOXP3 Controls an miR-146/NF-kappaB Negative Feedback Loop That Inhibits Apoptosis in Breast 
Cancer Cells. Cancer Res 2015; 75: 1703-13.

10. Poller W, Tank J, Skurk C, Gast M. Cardiovascular RNA interference therapy: the broadening tool and target spectrum. Circ Res 2013; 113: 588-602.

11. Yuan JH, Yang F, Wang F, Ma JZ, Guo YJ, Tao QF et al. A long noncoding RNA activated by TGF-beta promotes the invasion-metastasis cascade in hepatocellular carcinoma. Cancer Cell 2014; 25: 666-81.

12. Simon MD, Wang CI, Kharchenko PV, West JA, Chapman BA, Alekseyenko AA et al. The genomic binding sites of a noncoding RNA. Proc Natl Acad Sci U S A 2011; 108: 20497-502. 